\title{
Publisher's Note: Transient Casimir Forces from Quenches in Thermal and Active Matter [Phys. Rev. Lett. 118, 015702 (2017)]
}

\author{
Christian M. Rohwer, Mehran Kardar, and Matthias Krüger \\ (Received 18 January 2017; published 27 January 2017)
}

DOI: 10.1103/PhysRevLett.118.049904

This paper was published online on 5 January 2017 with an error in the text on page 1. On page 1, the beginning of the fourth sentence in the second paragraph in the right-hand column should read as "The former originates from the interactions among the microscopic constituents (including any obstacles)... ." The text has been corrected as of 19 January 2017. The text is incorrect in the printed version of the journal. 